\title{
Dielectric function and critical points of cubic and hexagonal CdSe
}

\author{
C. Janowitz \\ Paul Drude Institut für Festkörperelektronik, Hausvogteiplatz 5-7, D-10117 Berlin, Federal Republic of Germany \\ and Max Planck Institut für Festkörperforschung, Heisenbergstrasse 1, D-70569 Stuttgart, Federal Republic of Germany \\ O. Günther and G. Jungk \\ Paul Drude Institut für Festkörperelektronik, Hausvogteiplatz 5-7, D-10117 Berlin, Federal Republic of Germany \\ R. L. Johnson \\ Universität Hamburg, II. Institut für Experimentalphysik, Luruper Chausse 149, D-22603 Hamburg, Federal Republic of Germany \\ P. V. Santos and M. Cardona \\ Max Planck Institut für Festkörperforschung, Heisenbergstrasse 1, D-70569 Stuttgart, Federal Republic of Germany \\ W. Faschinger and H. Sitter \\ Johannes Kepler Universität Linz, Institut für Experimentalphysik, A-4040 Linz, Austria
}

(Received 28 January 1994)

\begin{abstract}
The dielectric function of cubic and hexagonal CdSe has been measured by spectroscopic ellipsometry in the 2-25-eV range. The results are compared with similar data for CdS and other II-VI compounds and also with band-structure calculations. Relevant critical points are listed. Transitions originating in the $\mathrm{Cd} 4 d$ levels are identified and an estimate of the corresponding exciton binding energies is made.
\end{abstract}

\section{INTRODUCTION}

A comparison of the physical properties of cubic zincblende and wurtzite phases of IIB-VIA semiconductors like CdS (Ref. 1) and CdSe is of interest for a number of reasons. Although in both modifications the arrangement of first and second neighbor atoms is very similar, ${ }^{2}$ recent investigations ${ }^{1}$ found their electronic excitation spectra to be significantly different. In the IIB column compounds the uppermost occupied cation $d$ levels lie in the lower portion of the valence band (VB). They are, therefore, a borderline case between III-V semiconductors, where the $d$ bands are well below the VB, and compounds with one element from the left side of the IIB column (copper, transition metals), where the $d$ bands lie near the Fermi energy. The $d$ levels should, therefore, hybridize somewhat with the valence bands ${ }^{3}$ and affect the electronic and bonding properties. A detailed knowledge of the electronic structure is also required for the application of these compounds in optoelectronic and electroacoustic devices.

A few early band-structure calculations for $\mathrm{CdSe}$ and $\mathrm{CdS}$ appeared mainly in the 60s (for references see Cohen and Chelikowsky ${ }^{4}$ ). More recent band calculations, like those of Chang, Froyen, and Cohen ${ }^{5}$ incorporate the $\mathrm{Cd}$ $4 d$ "semicore" levels in the VB and compare the band structures of the two modifications of CdS obtained with the local-density approximation (LDA) using the $a b$ initio pseudopotential method without spin-orbit (SO) interaction. The wurtzite modification of CdSe and CdS were treated by Kobayashi et al. ${ }^{6}$ with the semiempirical tight-binding (TB) method and most recently by $\mathrm{Xu}$ and Ching $^{7}$ with the orthogonalized linear combination of atomic-orbitals (OLCAO) method without SO coupling. The only study of the band structure of cubic CdSe available to us is a linear-muffin-tin-orbital (LMTO) LDA calculation by Christensen, ${ }^{8}$ who included SO effects and treated the Cd $4 d$ levels as bandlike states.

In this paper, we measured the dielectric function of CdSe in both modifications. The results are compared to similar ones obtained for CdSe (Ref. 1) and discussed on the basis of the calculated electronic band structures just mentioned. At energies below 10-eV structures due to the conventional interband transitions with initial states in the occupied $s p^{3}$ bands are seen. The structures above $10 \mathrm{eV}$ have as initial states mainly $\mathrm{Cd} 4 d$ levels and reflect the density of empty conduction states. Core exciton binding energies are estimated from these measurements and from the core-level binding energies found in the literature.

\section{EXPERIMENTAL METHOD}

Bulk samples of CdSe crystallize in the hexagonal close packed wurtzite structure. To obtain the face-centeredcubic zinc-blende modification, films of $5000 \AA$ [CdSe, lattice constant $a=6.05 \AA$ (Ref. 9)] thickness were grown by molecular-beam epitaxy (MBE) on a GaAs substrate (lattice constant $a=5.65 \AA$ ). The thickness of the latter film is so large that the large mismatch to the lattice constant of the substrate is expected to be relaxed to that of bulk CdSe. In fact, the structure of the cubic CdSe layers determined by $x$-ray diffraction yielded a lattice constant of $6.05 \AA$, as corresponds to fully relaxed CdSe. Hofmann et al. ${ }^{1}$ reported that an MBE-grown CdS film undergoes a phase transition to the hexagonal phase at a 
thickness of about $4500 \AA$. In a more recent publication Zahn et al. ${ }^{10}$ found this transition already occurring at $2200 \AA$. This transition shows up clearly in the ellipsometric spectra. A similar transformation is likely to occur for CdSe but has not been observed up to the thickness used in the present work (5000 $\AA$ ).

For the preparation of the two CdS structural modifications we refer to Hofmann et al. ${ }^{1}$ Single-crystal layers of CdSe with zinc-blende structure were grown at a rate of 1 monolayer/sec on a GaAs(100) substrate at a temperature of $300{ }^{\circ} \mathrm{C}$ in a MBE system described elsewhere. ${ }^{11}$ The sample was transferred under atmospheric pressure to the vacuum ultraviolet ellipsometer placed behind the 2-m Seya Namioka monochromator which utilizes the synchrotron radiation of the Berlin electron storage ring BESSY. Details of the optical system have been described previously. ${ }^{12}$ Prior to that, the crystals were investigated with a conventional rotating analyzer ellipsometer in the visible and UV range $(h v<5.5 \mathrm{eV})$. No significant differences with respect to the data obtained at BESSY in this energy region were found.

The results for wurtzite-type CdSe were obtained from well-polished single crystals with the $c$ axis in the surface plane. The so-called pseudodielectric function (i.e., including possible effects of surface layers different from bulk CdSe) along and perpendicular to the $c$ axis were obtained by analyzing the ellipsometric data using a uniaxial model for the wurtzite crystal. ${ }^{13}$ Linearly polarized light from the monochromator impinged without entrance polarizer directly on the sample, either parallel or perpendicular to the plane made up of the surface normal and the $c$ axis in the case of the wurtzite crystal. The ellipsometric parameters were measured with a rotating $\mathrm{MgF}_{2}$ Rochon analyzer below $9.5-\mathrm{eV}$ photon energy and with a triple-reflection gold polarizer in the energy region above $11 \mathrm{eV}$. Samples were held at $90 \mathrm{~K}$ during all measurements except the measurement at room temperature with the laboratory ellipsometer to enhance resolution.

\section{EXPERIMENTAL RESULTS}

In order to obtain from ellipsometric data the pseudodielectric functions of noncubic crystals it is often customary and convenient to use the approximation suggested by Aspnes. ${ }^{14}$ Within this approximation one processes the ellipsometric parameters as if the material were isotropic. The component of the pseudodielectric function $\epsilon(\omega)$ polarized in the plane of incidence is then obtained, provided the magnitude of $\epsilon$ is large compared to that of vacuum. While in our case this condition holds for $h v<8 \mathrm{eV}$, it does not at higher frequencies. We have therefore compared in Fig. 1 the data obtained by the Aspnes method, with $E$ perpendicular to the plane of incidence $(E \perp c)$ with those calculated using the full ellipsometric equation of the uniaxial crystal. ${ }^{15}$ We see that both methods yield indeed rather similar results for $h v<6.5 \mathrm{eV}$. For $h v>6.5 \mathrm{eV}$, however, differences are noticeable. These differences concern mainly the magnitudes of $\epsilon_{1}$ and $\epsilon_{2}$ : the characteristic spectral structures, which we assign below to critical points, are reproduced by both methods with almost the same shape and peak
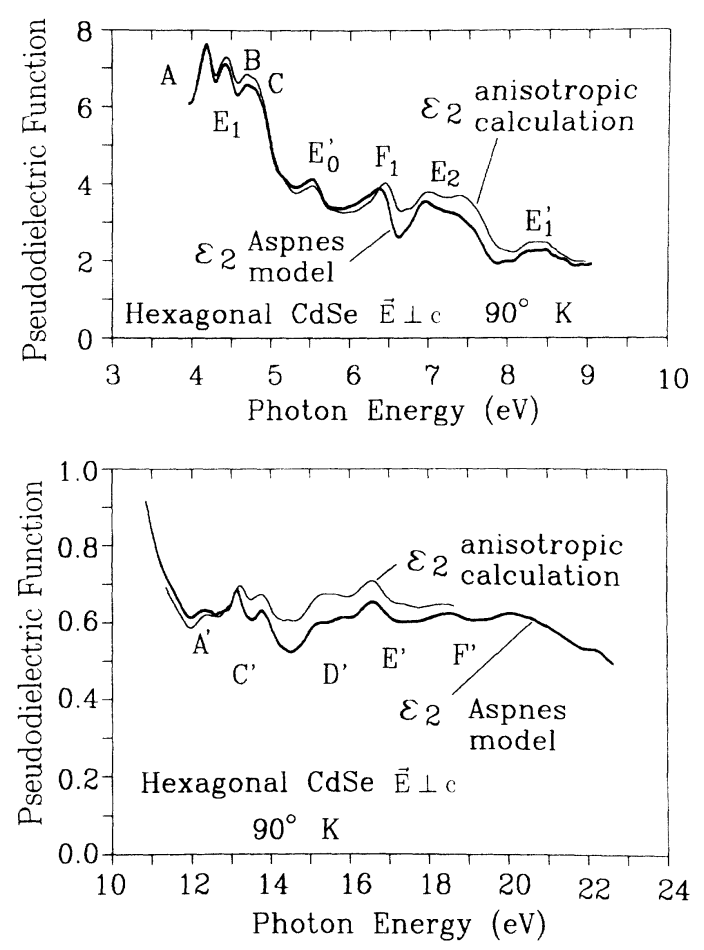

FIG. 1. Comparison of the pseudodielectric functions of hexagonal CdSe obtained by solving the full ellipsometric equations (thin lines) (Ref. 15) with these obtained with the approximate Aspnes method (thick lines) (Ref. 14) for an electric field polarized perpendicular to the $c$ axis.

energies. The differences between both types of curves are maximum in the region of the plasma frequency $(9-13 \mathrm{eV})$. Since no line shape analysis of the spectra will be performed, we will use for the discussion of critical point energies only the data obtained from the simpler Aspnes method.

Figure 2 shows the real and imaginary parts of the pseudodielectric constant of cubic and also of hexagonal CdSe for both polarization geometries in the region $1.5<h v<9 \mathrm{eV}$. The second derivative of $\epsilon_{2}(\omega)$ is also depicted for each set of spectra. The minima reproduce reasonably well the energy of most critical points. A more detailed critical point analysis would be rather arbitrary in view of the many structures observed.

\section{A. Cubic CdSe}

Concentrating first on the results for the fcc-CdSe, one sees that the overall shape of our spectra is similar to those reported by Hofmann et al. ${ }^{1}$ for cubic CdS, where from we have adopted the notation for the corresponding structures in CdSe. Differences occur in the $E_{1}$ region, where we were able to resolve the structures corresponding to the $E_{1}$ and $\left(E_{1}+\Delta_{1}\right)$ critical points both with synchrotron radiation and with a conventional rotating analyzer ellipsometer. These two peaks are not resolved for cubic $\mathrm{CdS},{ }^{1}$ a fact which is not surprising in view of the smaller spin-orbit splitting of sulfur as compared to Se.

As for many other cubic III-V and II-VI's semiconduc- 

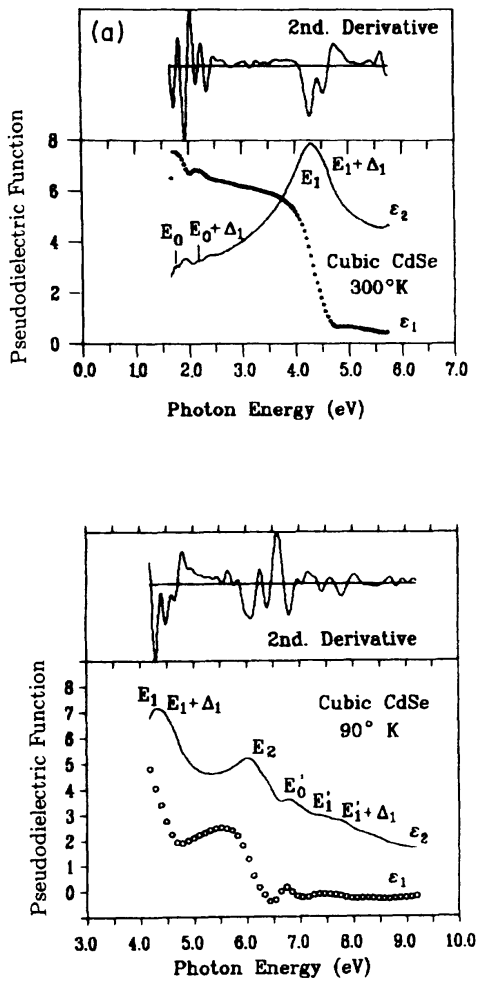

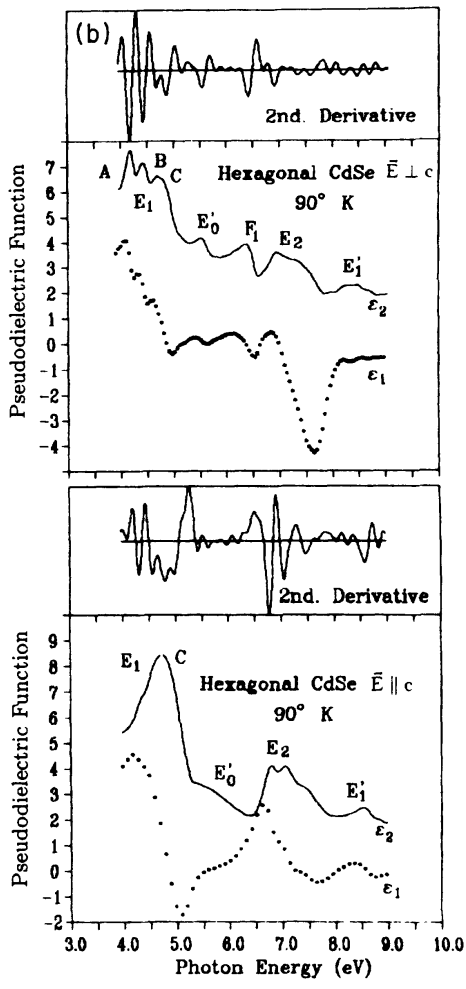

FIG. 2. (a) Pseudodielectric functions of cubic CdSe near the fundamental absorption edge measured at room temperature and in the region of the interband transitions at $90 \mathrm{~K}$. The upper curve displays the second derivative of the imaginary part of the pseudodielectric constant. In the energy region of the $E_{0}$ critical point the pseudodielectric function also includes contributions from the GaAs substrate. (b) Pseudodielectric functions of hexagonal CdSe in the region of the interband transitions at $90 \mathrm{~K}$. ( $\epsilon_{1}$ circles, $\epsilon_{2}$ solid line.) The upper inset of each figure shows the second derivative of $\epsilon_{2}$. tors the lowest band gap $\left(E_{0}\right)$ is direct and occurs at $\Gamma$. The energy of this critical point $\left(E_{0}=1.74 \mathrm{eV}\right.$ at room temperature) and of its spin-orbit split counterpart $\left(E_{0}+\Delta_{0}=2.15 \mathrm{eV}\right.$, yielding a spin-orbit splitting $\Delta_{0}=0.41 \mathrm{eV}$ ) in cubic CdSe was obtained from the minima in the second derivative of $\epsilon_{2}(\omega)$ displayed in Fig. 2(a). An experimental difficulty in the analysis of the second derivative spectra is the presence of interference fringes due to the finite thickness and low absorption coefficient of the CdSe cubic film for energies below 2.5 $\mathrm{eV}$. In this energy region, the pseudodielectric function displayed in Fig. 2(a) also includes contributions from the GaAs substrate. For a film thickness of $5000 \AA$, however, the structures from the interferences fringes are broader than those of the critical points and can be easily distinguished in the second derivative spectrum.

The dominant peaks in the $\epsilon(\omega)$ spectra at higher energies are the so-called $E_{1}, E_{2}$, and $E_{1}^{\prime}$ structures, which can be assigned to interband transitions. The $E_{1}$ structure is caused by transitions of electrons from the uppermost spin-orbit-split valence bands to the lowest conduction bands along the $\langle 111\rangle$ direction. The $E_{2}$ structure contains contributions from different regions of the Brillouin zone, mostly from the $\Sigma$ direction and in the $\Gamma-X$ $U-L$ plane. Their peak energy $E_{2}$ corresponds to the well-known average interband energy (often called the Penn gap).

We have listed in Table I the energies of the structures in the pseudodielectric function of cubic CdSe determined at $90 \mathrm{~K}$ from the second derivative spectrum as discussed above. For the sake of comparison we have also listed the corresponding energies for cubic CdS as reported in Ref. 1 and those for CdTe obtained by ellip- sometry ${ }^{16}$ and by reflectivity measurements with synchrotron radiation. ${ }^{17}$ We note that the critical-point energies for CdSe are overall about $0.5 \mathrm{eV}$ lower than for CdS. They are also systematically higher by about $1 \mathrm{eV}$ than those for CdTe, with the exception of the $E_{0}$ gap, which is only shifted by $0.1 \mathrm{eV}$.

Concerning the absolute values of $\epsilon$ and peak ratios in $\epsilon_{2}$ in comparison to prototype GaAs (Ref. 18) bandstructure calculations and reliable theoretical $\epsilon_{2}(\omega)$ curves would be desirable. At this point the sample quality of the artificially produced cubic modifications of CdSe and CdS must be discussed. Two effects, namely, strain from the lattice mismatch between substrate and the sample and also the possibility of nonideal crystallization (wurtzite-type domains) may affect the bulk electronic structure. We nevertheless think that these effects are only of minor importance: for layer thicknesses of 5000 $\AA$ and penetration depths of about $200 \AA$ the bulk lattice constant should have been recovered through production of misfit dislocations. The x-ray diffractometer data do not show any evidence of the wurtzite phase. Moreover, our $\epsilon_{2}$ spectra for cubic CdSe look rather similar to those of other II-VI compounds like CdTe (Refs. 19 and 26) which crystallize naturally in the zinc-blende structure. The ratio of the heights of the $E_{2}$ and $E_{1}$ peaks in $\epsilon_{2}(\omega)$ reported for CdTe in (Refs. 19 and 26) is similar in CdS and in CdSe. However, we must point out that much higher $E_{2} / E_{1}$ ratios were reported in Ref. 17 for CdTe as obtained by the Kramers and Kronig analysis of reflectivity data and that our data on $\mathrm{CdSe}$ may be affected by native oxide overlayers. Nevertheless this discrepancy of the peak ratios should be further investigated. 
TABLE I. Comparison between experimental and theoretical transition energies (in eV) of interband critical points in cubic CdTe, CdS, and CdSe. (a) Ref. 5, SC-LDA including $4 d$ levels, (b) Ref. 16, ellipsometry ( $T=25 \mathrm{~K}$ ), (c) Ref. 10, ellipsometry (room temperature), (d) ellipsometry (room temperature), (e) Ref. 8, relativistic LDA including $4 d$ levels, (f) Ref. 17, reflectivity ( $T=300 \mathrm{~K}$ ), (g) Ref. 1, ellipsometry $(T=90 \mathrm{~K})$, (h) ellipsometry $(T=90 \mathrm{~K})$.

\begin{tabular}{|c|c|c|c|c|c|c|}
\hline Transition & $\begin{array}{l}\text { Cubic } \\
\text { CdTe } \\
\text { theory }\end{array}$ & $\begin{array}{c}\text { Cubic } \\
\text { CdTe } \\
\text { experimental }\end{array}$ & $\begin{array}{l}\text { Cubic } \\
\text { CdS } \\
\text { theory }\end{array}$ & $\begin{array}{c}\text { Cubic } \\
\text { CdS } \\
\text { experimental }\end{array}$ & $\begin{array}{l}\text { Cubic } \\
\text { CdSe } \\
\text { theory }\end{array}$ & $\begin{array}{c}\text { Cubic } \\
\text { CdSe } \\
\text { experimental }\end{array}$ \\
\hline \multirow[t]{2}{*}{$E_{0}$} & $1.65^{\mathrm{a}}$ & $1.6^{\mathrm{b}}$ & $2.4^{\mathrm{a}}$ & $2.45^{c}$ & & $1.74^{\mathrm{d}}$ \\
\hline & $1.6^{\mathrm{e}}$ & $1.51^{\mathrm{f}}$ & $2.5^{\mathrm{e}}$ & & & \\
\hline$E_{0}+\Delta_{0}$ & & & & & & $2.15^{\mathrm{d}}$ \\
\hline \multirow[t]{2}{*}{$E_{0}^{\prime}$} & $5.7^{\mathrm{a}}$ & & $7.5^{\mathrm{a}}$ & $7.4^{\mathrm{g}}$ & $7.8^{\mathrm{e}}$ & $6.80^{\mathrm{h}}$ \\
\hline & $6.1^{\mathrm{e}}$ & & $8.4^{\mathrm{e}}$ & & & \\
\hline \multirow[t]{2}{*}{$E_{1}$} & $3.5^{\mathrm{a}}$ & $3.52^{\mathrm{b}}$ & & & $4.5^{\mathrm{e}}$ & $4.29^{\mathrm{h}}$ \\
\hline & $3.4^{\mathrm{e}}$ & $3.32^{\mathrm{f}}$ & & & & \\
\hline \multirow[t]{2}{*}{$E_{1}+\Delta_{1}$} & $4.0^{\mathrm{a}}$ & $4.08^{b}$ & $5.4^{\mathrm{a}}$ & $5.0^{g}$ & $4.8^{\mathrm{e}}$ & $4.49^{h}$ \\
\hline & $4.1^{\mathrm{e}}$ & $3.90^{f}$ & $5.0^{\mathrm{e}}$ & & & \\
\hline \multirow[t]{2}{*}{$E_{1}^{\prime}$} & $6.9^{\mathrm{a}}$ & $6.7^{f}$ & $9.1^{\mathrm{a}}$ & $8.3^{\mathrm{g}}$ & $8.9^{\mathrm{e}}$ & $7.40^{\mathrm{h}}$ \\
\hline & $7.2^{\mathrm{e}}$ & & $9.3^{\mathrm{e}}$ & & & \\
\hline \multirow[t]{2}{*}{$E_{1}^{\prime}+\Delta_{1}$} & & $7.8^{f}$ & & & $9.2^{\mathrm{e}}$ & $7.80^{\mathrm{h}}$ \\
\hline & $7.7^{\mathrm{e}}$ & & $9.5^{\mathrm{e}}$ & & & \\
\hline \multirow[t]{4}{*}{$E_{2}$} & $5.7^{\mathrm{a}}$ & & $7.1^{\mathrm{a}}$ & $6.4^{g}$ & $6.7^{\mathrm{e}}$ & $6.09^{h}$ \\
\hline & $5.6^{\mathrm{e}}$ & $5.3^{\mathrm{f}}$ & $6.8^{\mathrm{e}}$ & & & \\
\hline & $6.0^{\mathrm{a}}$ & & $8.2^{\mathrm{a}}$ & $6.9^{\mathrm{g}}$ & $7.4^{\mathrm{e}}$ & $6.39^{\mathrm{h}}$ \\
\hline & $6.0^{\mathrm{e}}$ & & $7.6^{\mathrm{e}}$ & & & \\
\hline
\end{tabular}

\section{B. Hexagonal CdSe}

We discuss next the pseudodielectric function of wurtzite-type CdSe [Fig. 2(b)]. Our experimental results for both polarizations are similar to these for CdS. ${ }^{1} \mathrm{We}$ have, therefore, labeled the structures in the same way as in (Ref. 1). The observed structures are polarization dependent and also differ from those observed in the cubic phase. In particular, no splitting of the $E_{1}$ peak is observed for the $E \| c$ configuration (C), whereas for electric fields perpendicular to the $c$ axis the $E_{1}$ structure splits into three peaks $(A, B$, and $C$ ), a fact which was early observed in reflection spectra. ${ }^{20}$ In order to understand the origin of the splitting, we reproduce in Fig. 3(a) the OLCAO band structure calculation of hexagonal CdSe of $\mathrm{Xu}$ and Ching. ${ }^{7}$ According to Logothetidis et al. ${ }^{13}$ and Cohen and Chelikovsky ${ }^{4}$ the $A$ and $B$ transitions correspond to excitation along the $\Gamma \boldsymbol{A}$ direction of the Brillouin zone [see Fig. 3(a)], which are only allowed for $E \perp c$. The extra transition $C$, observed at the same energy for both polarizations, is probably due to transitions along the lateral faces of the Brillouin zone ( $L M$ line). In Fig. 3(b) the measured pseudodielectric function for $E \| c$ (upper curve) and $E \perp c$ (lower curve) are compared with the theoretical results of Ref. 7. A reasonable agreement to the experimental data has been obtained by shifting the experimental data by $0.6 \mathrm{eV}$ to higher energies. In agreement with the previous discussion, the calculated $\epsilon_{2}$ curve also shows additional structures for $E \perp c$, similar to the experimental $A, B$, and $C$ structures, but not as pronounced.

The transitions above the $E_{1}$ critical point have contributions from different points of the Brillouin zone, mak- ing a detailed assignment to interband transitions obtained from band-structure calculations difficult. The assignment of these transitions in Fig. 2 was made by comparison with similar spectra for hexagonal CdS (Ref. 1) who adopted their notation from a calculation of the optical transition selection rules. ${ }^{21}$

\section{Core-level transitions}

The pseudodielectric function of both cubic and hexagonal CdSe in the photon energy range above $12 \mathrm{eV}$ (Fig. 4 ) is dominated by transitions with the semicore $\mathrm{Cd} 4 d$ states and the $s$-like band as initial states. The $\mathrm{Cd} 4 d$ states have been treated in more recent publications ${ }^{7,8}$ as bandlike states belonging to the valence band.

As in the case of cubic and hexagonal CdS (Ref. 1) the spectra of $\epsilon(\omega)$ of cubic and hexagonal (both $E \| c$ and $E \perp c)$ CdSe show a certain degree of similarity in the main features. These spectra are depicted in Fig. 4 together with the second derivative of $\epsilon_{2}(\omega)$ in the photon energy range $11<h v<23 \mathrm{eV}$. Common to all spectra is a small peak at the onset of the absorption around $12 \mathrm{eV}$, a group of transitions between 13 and $14.5 \mathrm{eV}$ (labeled $C^{\prime \prime}$ for cubic and $C^{\prime}$ for hexagonal $\mathrm{CdSe}$ ), and a third group starting at $15 \mathrm{eV}$ (labeled $D^{\prime \prime}$ for cubic and $D^{\prime}$ to $F^{\prime}$ for hexagonal CdSe). We assign the initial state of these transitions to $\mathrm{Cd} 4 d_{3 / 2}$ and $\mathrm{Cd} 4 d_{5 / 2}$ core levels. Shevchik et al. ${ }^{22}$ reported a value of 9.55 and $10.28 \mathrm{eV}$ below the top of the valence band for the $\mathrm{Cd} 4 d_{5 / 2}$ and $4 d_{3 / 2}$ levels of polycrystalline material, Magnusson et al. ${ }^{23}$ gave a value of $10.3 \mathrm{eV}$ for the weighted average binding energy of the $\mathrm{Cd} 4 d$ levels in wurtzite CdSe. The $\mathrm{Cd} 4 d$ levels are expected to be almost dispersionless. ${ }^{8}$ For comparison, the 

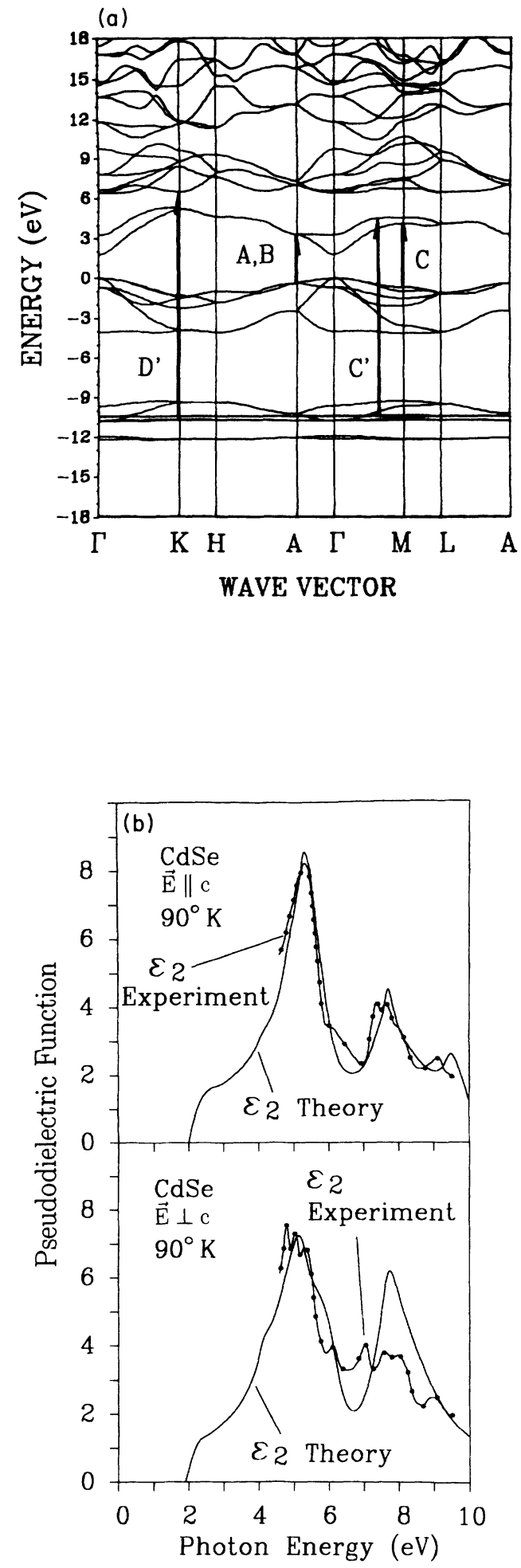

FIG. 3. (a) OLCAO band structure for CdSe (Ref. 7). The vertical lines denote optical transitions observed in the ellipsometric spectra. (b) Comparison between the calculated imaginary part of the pseudodielectric function (drawn lines) along the $c$ axis $\left(\epsilon_{2 x y}\right)$ and perpendicular to it $\left(\epsilon_{2 x}\right)$ (from Ref. 7) with the experimental values (lines with dots) obtained in the $E$ parallel to $c$ and in the $E$ perpendicular to $c$ geometries from solving the full anisotropic equations (Ref. 15). The experimental values were shifted by $0.6 \mathrm{eV}$ towards higher energies to achieve agreement between experiment and theory. dispersion of the Cd4d states in electronically similar cubic CdTe was found to be only $0.12 \mathrm{eV}$ along the $\Gamma L$ line. ${ }^{24}$

The weak structure near $12 \mathrm{eV}$ is assigned to final states around the lowest conduction-band point (at $\Gamma$ ). While $d \rightarrow s$ transitions are forbidden, admixture of $p$ to the $s$ conduction states around $\Gamma$, or the $f$ admixture which should occur in the $\Gamma_{1}$ states, could make the transitions weakly allowed.

The average experimental binding energy of $10.3 \mathrm{eV}$ (Refs. 22 and 23) is lower than the 10.5-12 eV prediction

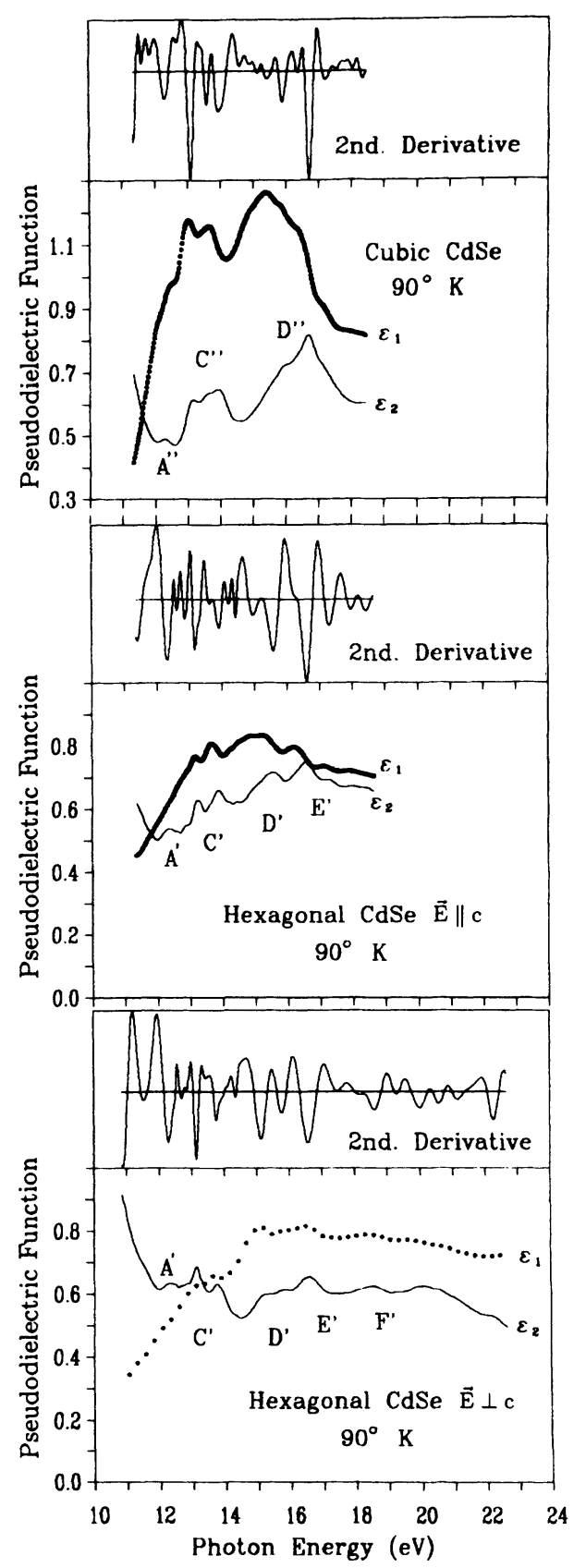

FIG. 4. Dielectric functions of cubic (upper panel) and hexagonal CdSe (lower panels) in the region of the $4 d$ transitions at $90 \mathrm{~K}$. ( $\epsilon_{1}$ circles, $\epsilon_{2}$ solid line). The upper inset of each figure shows the second derivative of $\epsilon_{2}$. 
of $\mathrm{Xu}$ and $\mathrm{Ching}^{7}$ for hexagonal CdSe and their $4 d$ core states are energetically below the $s$ levels. Christensen, on the contrary, found in LDA-LMTO calculations the Cd $4 d$ levels to be energetically above the $s$ levels, at a binding energy of 7.5-7.8 eV $\left(\mathrm{Cd} 4 d_{5 / 2}\right), 8.29 \mathrm{eV}$ $\left(\mathrm{Cd} 4 d_{3 / 2}\right)$. Similar differences are encountered for most II-VI and III-V semiconductors. They are due to different relaxation effects for holes in $p$ like valence states and in $d$-like core states. The high binding energy of the $p$ states in the tight-binding calculation is probably due to having neglected relativistic effects in Ref. 7. These effects tend to increase the binding energy of the $s$ and $p$ states relative to the $d$ states. The reason why a larger binding energy for the core $d$ states (referred to the top of the valence band) is found experimentally than in relativistic LDA calculations can be attributed to the strong localization of the core states when compared to the predominantly $p$-like conduction states. A simple way to estimate relaxation energies (not included in the LDA treatment) is by using the so-called transition state, i.e., by calculating the change in total energy produced by a unit change in electron charge for a ground state with half an electron missing. ${ }^{25}$ For the localized $d$ electrons the use of the $+\frac{1}{2}$ transition state as ground state strongly reduces the effects of the Hartree-Fock energy while for the delocalized valence electrons this contribution is not changed by the use of the transition state. This raises the energy of the state with a hole in the $d$ shell with respect to a hole at the top of the valence band.

The second and third group of transitions $\left(C^{\prime}\right.$ and $\left.F^{\prime}\right)$ have final states in the group of conduction bands with energies between 3 and $5 \mathrm{eV}$ (transition $C^{\prime}$ ) and between 6 and $9 \mathrm{eV}$ (transitions $D^{\prime}$ to $F^{\prime}$ ), respectively [see Fig. 3(a)]. Xu and Ching ${ }^{7}$ calculated the unoccupied density of states (DOS) for these conduction bands. The maxima in this theoretical, unfilled DOS matches well with the inverse photoemission results of Ref. 23. The labels $C^{\prime}, D^{\prime}$, and $E^{\prime}$ used to identify the transitions in Fig. 4 correspond to those used to denote the maxima in the unoccupied DOS determined by inverse photoemission in Fig. 7 of Ref. 23. The two groups of conduction bands are separated by an energy gap of $1.5-2 \mathrm{eV}$ [see Fig. 3(a)]. Evidence for the existence of this gap is found in the dip in $\epsilon_{2}(\omega)$ centered at $14.6 \mathrm{eV}$. The calculated DOS of Xu and Ching ${ }^{7}$ shows a gap at $5.5-\mathrm{eV}$ binding energy between the first two groups of unoccupied bands. We also observe in the corresponding region of $h v=14.3 \mathrm{eV}$ only very weak structures for hexagonal $\mathrm{CdSe}$, whose origin is unclear.

The splitting between the $\mathrm{Cd} 4 d_{3 / 2}$ and $\mathrm{Cd} 4 d_{5 / 2}$ core levels gives rise to double peaked structures in the two transition groups described above. The transition energies determined from the minima of the second derivative of $\epsilon_{2}(\omega)$ and the corresponding splittings are summarized in columns 4 and 5 of Table II. The splitting of $0.62-0.74 \mathrm{eV}$ measured for the $C^{\prime}$ structure compares well with the $0.73 \mathrm{eV}$ splitting measured by Shevchik et al. ${ }^{22}$ in polycrystalline CdSe.

The splitting of $0.73 \mathrm{eV}$ is well reproduced in the LMTO band-structure calculation for CdSe by Christen$\operatorname{sen}^{8}$ which yields spin-orbit and crystal-field splittings of 0.76 and $0.27 \mathrm{eV}$, respectively, at the $\Gamma$ point. Note that the TB calculation of Fig. 3(a) cannot account for this splitting since it does not include spin effects. The splitting of the bands in this figure is due to orbital crystalfield effects.

Finally, the excitonic binding energies for the Cd $4 d$ transitions can be determined by comparing the ellipsometric transition energies with the core-level binding energies measured with photoemission ${ }^{22}$ and with the final state energies from inverse photoemission. We neglect in this analysis the dispersion of the core levels. We used the inverse photoemission results of Magnusson et $a l .{ }^{23}$ who gave the energy position relative to the

TABLE II. Excitonic transition and binding energies (for details, see text).

\begin{tabular}{|c|c|c|c|c|c|c|c|}
\hline Phase & Polarization & Label & & $\begin{array}{c}\text { Excitonic } \\
\text { transition } \\
\text { energy } \\
(\mathrm{eV})\end{array}$ & $\begin{array}{c}\text { SO } \\
\text { splitting } \\
(\mathrm{eV})\end{array}$ & $\begin{array}{c}\text { Binding } \\
\text { energy } \\
(\mathrm{eV}) \\
\text { Ref. } 4\end{array}$ & $\begin{array}{c}\text { Res. } \\
\text { Excitonic } \\
\text { shift } \\
(\mathrm{eV})\end{array}$ \\
\hline \multirow[t]{2}{*}{ Hex. } & $E \| c$ & $C^{\prime}$ & $d_{5 / 2}$ & 13.18 & 0.74 & 4.5 & 0.87 \\
\hline & & & $d_{3 / 2}$ & 13.92 & & & 0.86 \\
\hline \multirow[t]{2}{*}{ Hex. } & $E \| c$ & $D^{\prime}$ & $d_{5 / 2}$ & 14.95 & 0.65 & 6.3 & 0.90 \\
\hline & & & $d_{3 / 2}$ & 15.60 & & & 0.98 \\
\hline \multirow[t]{2}{*}{ Hex. } & $E \| c$ & $E^{\prime}$ & $d_{5 / 2}$ & 16.60 & 0.70 & 7.5 & 0.45 \\
\hline & & & $d_{3 / 2}$ & 17.30 & & & 0.48 \\
\hline \multirow[t]{2}{*}{ Hex. } & $E \perp c$ & $C^{\prime}$ & $d_{5 / 2}$ & 13.14 & 0.62 & 4.5 & 0.91 \\
\hline & & & $d_{3 / 2}$ & 13.76 & & & 1.02 \\
\hline \multirow[t]{2}{*}{ Hex. } & $E \perp c$ & $D^{\prime}$ & $d_{5 / 2}$ & 15.15 & 0.65 & 6.3 & 0.70 \\
\hline & & & $d_{3 / 2}$ & 15.80 & & & 0.78 \\
\hline \multirow[t]{2}{*}{ Hex. } & $E \perp c$ & $E^{\prime}$ & $d_{5 / 2}$ & 16.60 & 0.85 & 7.5 & 0.45 \\
\hline & & & $d_{3 / 2}$ & (17.45) & & & $(0.33)$ \\
\hline \multirow[t]{2}{*}{ Cubic } & & $C^{\prime \prime}$ & $d_{5 / 2}$ & 13.10 & 0.85 & & \\
\hline & & & $d_{3 / 2}$ & 13.95 & & & \\
\hline \multirow[t]{2}{*}{ Cubic } & & $D^{\prime \prime}$ & $d_{5 / 2}$ & 15.88 & 0.83 & & \\
\hline & & & $d_{3 / 2}$ & 16.70 & & & \\
\hline
\end{tabular}


valence-band maximum of several unoccupied, nearly flat bands along $\Gamma M\left(M_{1}, M_{4}, M_{3}\right)$, labeled $C^{\prime}, D^{\prime}$, and $E^{\prime}$ in (Ref. 23) (see column 6 of Table II). The $C^{\prime}$ transition have final states $4.5 \mathrm{eV}$ above the valence-band maxima. From the $9.55 \mathrm{eV}$ binding energy of the $\mathrm{Cd} 4 d_{5 / 2}$ core levels ${ }^{22}$ an energy of $14.05 \mathrm{eV}$ is expected for the $\mathrm{Cd}$ $4 d_{5 / 2} \rightarrow C^{\prime}$ transition in the absence of excitonic effects. The lower transition energy of $13.18 \mathrm{eV}$ measured by ellipsometry (see Table II) yields, therefore, an excitonic shift of $0.87 \mathrm{eV}$ for this transition. Table II summarizes the $4 d$ exciton energies for the different transitions observed for hexagonal CdSe. The fourth double structure labeled $F^{\prime}$, at 18.60 and $19.30 \mathrm{eV}$, is not incorporated in Table II. Relating it to the structure at a binding energy of $8.7 \mathrm{eV}$ from Magnusson ${ }^{23}$ gives an excitonic binding energy of $0.35 \mathrm{eV}$ for structure $F^{\prime}$. Since experimental data for the binding energies and final-state densities are not available for the cubic phase, the excitonic shifts could not be determined for this modification. However, they should be rather similar to those found for the wurtzite modification.

To check the excitonic contribution to the weak structure $A^{\prime}$, respectively, $A^{\prime \prime}$ having its onset at $12 \mathrm{eV}$ and its minimum in the 2 nd derivative at $12.3 \mathrm{eV}$ one must mention first, that it is rather broad and lacks a clearly resolved spin-orbit split counterpart, even in the 2nd derivative spectra. Furthermore, taking the simple mean value of $(9.55 \mathrm{eV}+10.28 \mathrm{eV}) / 2=9.9 \mathrm{eV}$ of Ref. 22 as the average binding energy of the $4 d$ core levels and using the room temperature value of $1.74 \mathrm{eV}$ for the fundamental gap of cubic and $1.83 \mathrm{eV}$ as the lowest band-gap energy at $15 \mathrm{~K}$ in hexagonal CdSe (Ref. 13) we arrive at an energy of $(9.9 \mathrm{eV}+1.74 \mathrm{eV})-12.3 \mathrm{eV}=-0.66 \mathrm{eV}$ for cubic and $-0.57 \mathrm{eV}$ for hexagonal $\mathrm{CdSe}$, i.e., binding energies with the wrong sign. The reason for that lies in the very low state density at the conduction-band minimum, which makes it plausible that the $A^{\prime}$ and $A^{\prime \prime}$ peaks are due to transitions into slightly higher parts of the CB. Note that at the $\Gamma$ point the transitions are nearly forbidden as discussed above. Generally we expect because of the small effective mass of the lowest $C B$ at $\Gamma$ a very small excitonic binding energy, while for the higher transitions $C^{\prime}$ and $D^{\prime}$ average excitonic binding energies of $0.9 \mathrm{eV}$ and for $E^{\prime} 0.4 \mathrm{eV}$ have been observed.

\section{CONCLUSION}

We have performed ellipsometric measurements of the optical functions of cubic and hexagonal CdSe using radiation of the storage ring BESSY. We have identified the interband critical points for the cubic phase in the region $h v<10 \mathrm{eV}$ and assigned them to the results of bandstructure calculations. These results have been compared with corresponding data measured earlier for CdTe and cubic CdS. The optical functions of hexagonal CdSe have also been compared with the results of band-structure calculations. In the region $h v>12 \mathrm{eV}$ transitions originating at the $4 d$ core levels of $\mathrm{Cd}$ are observed. They are grouped in doublets which correspond to the spin-orbit split $d_{5 / 2}-d_{3 / 2}$ states. The corresponding peaks seem to be of excitonic (i.e., localized) nature. An estimate of the exciton binding energy has been performed.

\section{ACKNOWLEDGMENTS}

We acknowledge N.E. Christensen for making available to us his unpublished band-structure calculations and $\mathbf{M}$. Kuball for a critical reading of the manuscript. We thank O. Gunnarson for discussion on the role of relaxation on the binding energy of core electrons. This work was funded partially by the Bundesminister für Forschung und Technologie (05 5ESCAB 9).
${ }^{1}$ Ph. Hofmann, K. Horn, A. M. Bradshaw, R. L. Johnson, D. Fuchs, and M. Cardona, Phys. Rev. B 47, 1639 (1993).

2J. L. Birman, Phys. Rev. 115, 1493 (1959).

${ }^{3}$ N. E. Christensen, Phys. Rev. B 37, 4528 (1988); S. H. Wei and A. Zunger, ibid. 37, 8958 (1988).

${ }^{4}$ M. L. Cohen and J. R. Chelikowsky, Electronic Structure and Optical Properties of Semiconductors (Springer, Heidelberg, 1988).

${ }^{5}$ K. J. Chang, S. Froyen, and M. L. Cohen, Phys. Rev. B 28, 4736 (1983).

${ }^{6}$ A. Kobayashi, O. F. Sankey, S. M. Volz, and J. D. Dow, Phys. Rev. B 28, 935 (1983).

${ }^{7}$ Y. N. Xu and W. Y. Ching, Phys. Rev. B 48, 4335 (1993).

${ }^{8}$ N. E. Christensen (private communication).

${ }^{9}$ S. M. Sze, Physics of Semiconductor Devices, 2nd ed. (Wiley, New York, 1981), p. 848.

${ }^{10}$ D. R. T. Zahn, G. Kudlek, U. Rossow, A. Hoffmann, I. Broser, and W. Richter, Adv. Mat. Opt. Electron. 2, 11 (1993).

${ }^{11}$ W. Faschinger, P. Juza, S. Ferreira, H. Zajicek, A. Pesek, and H. Sitter, Thin Solid Films 225, 270 (1993).

${ }^{12}$ R. L. Johnson, J. Barth, M. Cardona, D. Fuchs, and A. M. Bradshaw, Nucl. Instrum. Methods A 290, 606 (1990).

${ }^{13}$ S. Logothetidis, M. Cardona, P. Lautenschlager, and M. Gar- riga, Phys. Rev. B 34, 2458 (1986).

${ }^{14}$ D. E. Aspnes, J. Opt. Soc. Am. 70, 1275 (1980).

${ }^{15}$ P. Drude, Wiedemann Ann. 32, 584 (1887); R. M. A. Azzam and N. M. Bashara, Ellipsometry and Polarized Light (NorthHolland, Amsterdam, 1977).

${ }^{16}$ D. T. F. Marple and H. Ehrenreich, Phys. Rev. Lett. 8, 87 (1962).

${ }^{17}$ J. L. Freeouf, Phys. Rev. B 7, 3810 (1973).

${ }^{18}$ D. E. Aspnes and A. A. Studna, Phys. Rev. B 27, 985 (1983).

${ }^{19}$ J. Barth, R. L. Johnson, and M. Cardona, Handbook of Optical Constants of Solids II (Academic, New York, 1991), p. 213.

${ }^{20}$ M. Cardona, Solid State Commun. 5, 109 (1962).

${ }^{21}$ M. A. Mojumder, Solid State Commun. 43, 13 (1982).

${ }^{22}$ N. J. Shevchik, J. Tejeda, M. Cardona, and D. W. Langer, Phys. Status Solidi B 59, 87 (1973).

${ }^{23}$ K. O. Magnusson, U. O. Karlsson, D. Straub, S. A. Flodström, and F. J. Himpsel, Phys. Rev. B 36, 6566 (1987).

${ }^{24}$ C. Janowitz, Doctoral thesis, Unversity of Kiel, 1991.

${ }^{25}$ M. Cardona and L. Ley, Photoemission in Solids I (SpringerVerlag, Heidelberg, 1978), p. 66.

${ }^{26}$ P. Lautenschlager, S. Logothetidis, L. Vina, and M. Cardona, Phys. Rev. B 32, 3811 (1985). 